\title{
Performance Evaluation of Routing Protocols on the Reference Region Group Mobility Model for MANET
}

\author{
Yan Zhang, Chor Ping Low, Jim Mee Ng \\ School of Electrical and Electronic Engineering, Nanyang Technological University, Singapore \\ E-mail: \{zh0003an,icplow\}@ntu.edu.sg \\ Received January 31, 2011; revised February 23, 2011; accepted February 28, 2011
}

\begin{abstract}
Group mobility is prevalent in many mobile ad hoc network (MANET) applications, such as disaster recovery, military operations, searching and rescue activities. Group partition, as an inherent phenomenon in group mobility, may occur when mobile nodes move in diverse mobility patterns and it causes the network to be partitioned into disconnected components. It may result in severe link disconnections, which interrupts network communications. To address this concern, we proposed a novel group mobility model in this paper, namely the Reference Region Group Mobility model, which can be used to mimic group operations in MANETs, i.e. group partitions and mergers. Based on this model, a comprehensive study on the impact of group partitions to the performance of network routing protocols are carried out by evaluating two well-known routing protocols, namely the Ad Hoc On-demand Distance Vector Routing protocol (AODV) and the Dynamic Source Routing protocol (DSR). The simulation results reflect that group partitions have a significant impact to the performance of network routing protocols.
\end{abstract}

Keywords: Ad Hoc Network, Group Mobility Model, Group Partition, Routing, AODV, DSR

\section{Introduction}

Mobility models are used in simulation studies to describe the dynamic behaviors of mobile devices in the real world for analyzing and evaluating the performance of ad hoc network protocols under various scenarios [1]. Mobility models play a significant role in the development of MANETs. Most existing mobility models, such as the Random Waypoint Mobility model [2] and the Random Walk Mobility model [3], are designed to simulate the movement of each individual, which are referred to as entity mobility models [4]. However, with the emergence of group-oriented applications, several group mobility models have been recently proposed. The applications requiring group mobility can be found in various scenarios which include military operations, searching and rescue in disaster recovery, visiting an exhibition hall, and firefighters operating in a building. The common characteristic of the above applications is that mobile nodes can be organized in the unit of groups, which could be further partitioned into many subgroups or merged with other groups. However, among all the existing group mobility models, none of them can simulate the inherent group operations, i.e., partitions and group mergers which are very common in most practical group mobility related scenarios. In addition, some group mobility models can only be applied to specific scenarios with the restrictions in the aspects of, e.g., fixed group membership, fixed velocity, and predefined paths for group's movement. By considering these restrictions, most of existing models are unable to describe the behaviors of group mobility realistically.

To address this issue, we proposed a novel mobility model in this paper, namely the Reference Region Group Mobility (RRGM) model. This model is a generic and parameterized mobility model which is able to model groups' movement. The novelty of this model is its ability to mimic group operations, such as group partitions and mergers. In addition, we introduce the concept of node density of a group. With a fixed number of group members, node density can be used to control the coverage area of a group (the range of an area that group members move within). Unlike existing group mobility models, RRGM allows mobile nodes to move independently without relying on the coordination of group leaders. By taking advantage of this, group partitions and mergers become possible.

Network routing protocols, as an important research 
topic in MANETs, have gained a lot of interest among the research community. A network routing protocol is used to exchange data packets between network users. In MANETs, each node learns about nodes nearby and how to reach them, and may also announce that it can reach them. Such discovery mechanisms allow routing information to be exchanged among all mobile nodes. Mobility patterns have an impact on the performance of network routing protocols, which has been discussed in some previous research works [5-7]. However, most existing works for studying the performance of routing protocols uses the entity mobility models which describe individual's mobility behaviors. On the other hand, we note that limited studies have been done on the impact of group mobility on network performance and they may usually assume that the network is connected, i.e. there are no group partitions and mergers. With the aid of RRGM model, we will evaluate and compare the performance of two well-known network routing protocols, namely Ad Hoc On-demand Distance Vector Routing (AODV) [8] and Dynamic Source Routing protocol (DSR) [9], under a network with the occurrence of group partitions and mergers in group mobility.

\section{Literature Review}

In this section, we will review some of the mobility models for MANETs first. Following that, review of the network routing protocols in MANETs will be presented.

\subsection{Review on Mobility Models in MANETs}

Mobility models in MANETs are generally classified into two categories, namely entity mobility models and group mobility models. Entity mobility models are used to describe the mobility of each individual's mobility while group mobility models mimic the movement of groups in MANETs.

The Random Waypoint Mobility model (RWP) [2] is a well-known entity mobility model. In this model, each mobile node randomly chooses a point as the destination and moves towards it with a randomly selected speed which is uniformly distributed in a range of $\left[V_{\min }, V_{\max }\right]$. After reaching the destination, the node may be stationary for a moment before generating a new destination. This process is repeated until the simulation ends.

Several group mobility models are designed for MANETs, although they may not be as widely used as entity mobility models. Reference Point Group Mobility (RPGM) model [10] is a generic group mobility model. In this model, the movement path of a group is predefined by a series of points which are referred to as "reference points”. Each group has a group leader which serves as the logical center of the group. Every mobile node follows the movement of the logical center with a random deviation in its position to that of the logical center. It is compulsory to predefine group membership and group leaders before running a simulation, which are not allowed to change during the simulation.

We note that group partitions and mergers could take place as a result of group mobility in MANETs. However, the existing models assume the membership of each group does not change which in turn does not allow mobile nodes to partition from groups or merge into other groups. We will address this issue in this paper.

\subsection{Review on Network Routing Protocols}

In ad hoc networks, routing protocols are typically categorized into two classes, table-driven routing protocols and on-demand routing protocols [11,12]. The two classes of routing protocols are differentiated by the mechanisms which they use to maintain and update routes in ad hoc networks [6,13-15]. In table-driven routing protocols, when a source has a packet to send, the routing information will be available immediately from its routing table which is updated periodically by advertisements, e.g. hello messages. However, in on-demand routing protocols, the source, which wants to send a packet, has to trigger a route discovery process if it can not find any fresh enough route from its routing table or the routes in its routing table are no longer available, and thus the routing information is updated by request. Both table-driven and on-demand routing protocols use more control overhead than the traditional static networks. In dynamic network environments such as MANETs, fast change of network topology will result in massive routing overhead generated to update routing tables of each mobile node, especially for the nodes using table-driven routing protocols. Table driven routing protocols are not adaptive to fast changes of network topology. On the other hand, on-demand routing protocols only need to update their routing information when they have packets to deliver. Hence, on-demand routing protocols generally outperform table-driven routing protocols in dynamic network environment. Thus, we choose two well-known on-demand routing protocols, i.e. AODV and DSR, for the study of the impact of group partitions and mergers on the network performance in this work. Next, we will review these two routing protocols.

"AODV minimizes the number of required broadcasts by creating routes on a demand basis” [8,14]. In AODV, when a source node desires to send a packet but does not have a valid path to the destination, it initiates a route discovery process to locate the destination by broadcasting a route request (RREQ) message to its neighbors, 
which then forward the request to their neighbors and so on, until either the destination or an intermediate node with a "fresh enough" route to the destination is located. Each node that forwards the RREQ creates a reverse route for itself back to the source node. The routing table is updated with the address of the neighbor from which the first copy of the broadcast message is received; thereby the reverse routes are established. Other additional copies of the same RREQ arrived later are discarded. The destination or any intermediate node with a "fresh enough" route to the destination responds by unicasting a route reply (RREP) packet back to the neighbor from which it first received the RREQ. The RREP is routed back along the reverse path hop-by-hop. The intermediate nodes update their route tables with the node from which the RREP is received as forward route entries. If an intermediate node moves, its upstream neighbors notices it and sends a link failure notification message to all its upstream neighbors to inform them of deletion of that route. The link failure notification message is relayed to the source which will choose to re-initiate a new route discovery process or discard.

DSR is a source-routed on-demand routing protocol [9]. In DSR, a node maintains route cache containing the source routes that it is aware of and updates entries in the route cache when it learns about new routes. The protocol consists of two major phases: route discovery and route maintenance. The route discovery phase is initiated by broadcasting a route request (RREQ) when the source node does not find a route to the destination in its route cache or if the route has expired. This RREQ contains the address of the destination, along with the source nodes' address and a unique identification number. To limit the number of RREQs propagated, a node processes the RREQ only if it has not already seen it before. Each node receiving the RREQ checks whether it knows of a route to the destination. If it does not, it adds its own address to the route record of the packet and then forwards the packet along its outgoing links. A route reply (RREP) is generated when either the destination or an intermediate node with current information about the destination receives the RREQ. In the route maintenance phase, each node transmitting the packet is responsible for confirming that the packet has been received by the next hop along the source route. Hello message is used to maintain the local connectivity of a node. By periodically broadcasting a hello message, a node may determine whether the next hop is within communication range. If no hello message is received, the node returns a route error (RRER) message to the original sender of the packet which can send the packet using another existing route or perform a new route discovery and remove the expired route information from its routing table.

Both AODV and DSR protocols employ a route dis- covery procedure. However, they have several important distinctions between each other. The most notable of these is that DSR uses more overhead in route constructions and route maintenance since each packet in DSR keeps much more routing information than that of AODV, whereas in AODV packets only contain the destination and source address. DSR is intended for networks in which the mobile nodes move at a moderate speed and the network is relatively small $[9,16]$. Additionally, DSR allows nodes to keep multiple routes to a destination in their route cache $[12,17]$. When a link on a route is broken, the source node can check its route cache for another route. However, DSR does not contain any mechanism to validate route entries when it faces with a choice of multiple routes. This leads to stale route entries, particular at high mobility environment. On the other hand, AODV allows nodes to keep only one route entry to each destination in the cache. The route discovery process will be reinitiated if the route in the route table of the source node is invalid.

\section{The Reference Region Group Mobility Model (RRGM)}

\subsection{Overview}

In this section, we will give an overview of our proposed mobility model that can be used to simulate group partitions and group mergers, namely the Reference Region Group Mobility model (RRGM). The generic case of group mobility in MANETs will be discussed where group partitions will be triggered by the events of generating new destinations. This can model the scenarios when a new task is assigned to a group, such as in a military operation or in search and rescue operations in disaster recovery. In the following parts of this section, we will introduce the group mobility in RRGM by different cases: a group assigned with a single destination and a group assigned with multiple destinations. In addition, group operations in RRGM, i.e. group partitions and group mergers, will also be described.

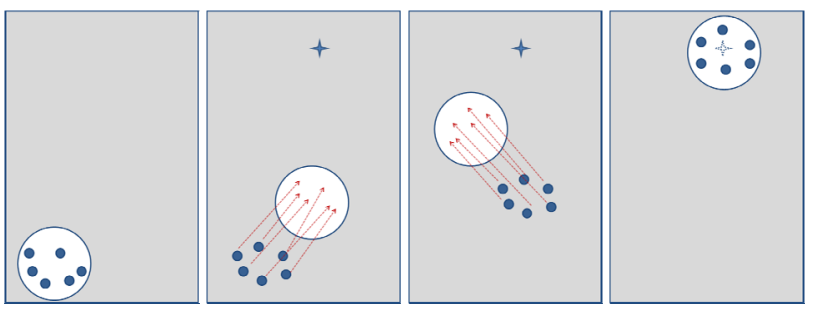

(a)

(b)

(c)

(d)

Figure 1. Mobility of a group (with a single destination). 
RRGM uses a novel concept, namely reference region, which is a dynamic area associated with a group of nodes. Location of a reference region changes dynamically during the simulation. The size of a reference region is determined by the number of nodes which are associated with it. Next, we will introduce the idea of reference region and how it is used by a group to move towards its destination in RRGM.

We bring out the idea of reference region and introduce the mechanism of RRGM model with a basic case first where a mobile group is assigned with a single destination. Initially, a group of mobile nodes are deployed in the simulation area and an area is created as the so-called "reference region" (the white circle in the figures) for this group as shown in Figure 1(a). Next, a destination is generated and it is assigned to the group which was created previously as shown in Figure 1(b); subsequently, a new reference region is generated in an area between the group and the destination (the location of the reference region and how to determine its size will be introduced in the next section). Assuming that every mobile node has the knowledge of the location of the reference region of its group, each of them randomly selects a point within the new reference region as its target and will move towards it. Later on, after all mobile nodes arrive at their respective targets in the reference region, the reference region will be relocated to a new area which is closer to the destination as illustrated by Figure 1(c). In this way, the reference region is iteratively relocated such that it is closer to the destination after each iteration. This process will stop when the destination falls within the most recent reference region being generated as shown in Figure 1(d). Finally, all mobile nodes arrived at the destination, after which the destination is removed to indicate the arrival of the group. When mobile nodes arrive at the destination, they may either pause for some time or continue their movement within the reference region. A node can continue its movement by randomly selecting a new point within the reference region.

In group mobility of MANETs, it is possible for a group to be assigned with multiple destinations simulta neously. When that happens, a group partition will take

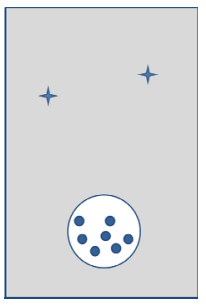

(a)

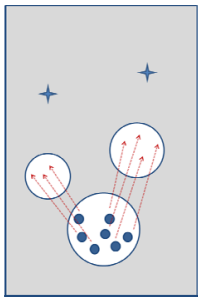

(b)

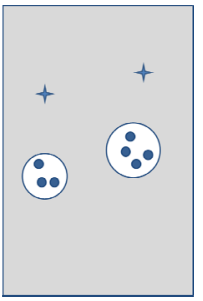

(c)
Figure 2. Mobility of a group (with multiple destinations). place as illustrated by Figure 2(a) to Figure 2(c). Initially, a group of mobile nodes are created and deployed into the simulation area, after which two destinations are generated simultaneously and assigned to the group as displayed in Figure 2(a). Correspondingly, two reference regions are created and each of them is placed between the group and one of the destinations respectively. Every mobile node in the original group randomly selects a point within either reference region as its target as shown in Figure 2(b). Finally, each mobile node moves to their respective targets in the corresponding reference regions as shown in Figure 2(c). The reference regions are iteratively relocated such that it is closer to the destination after each iteration and eventually mobile nodes will arrive at their respective destinations.

Reference region is also used for mergers between groups in RRGM. Practically, it is likely for a small group to merge with a larger group in MANETs. We assume that every mobile node has the knowledge of the location of all the groups, the location of the reference region of its group's, and the number of nodes in each group during a simulation. Based on this information, mobile nodes in a standby group can calculate the distance between its group and other groups respectively. The closest group, which has the smallest distance to the standby group, will be selected as the target for the standby group to merge with. As displayed in Figure 3(a), there are two groups in the simulation area of which one group is bigger than the other. In RRGM, for a group not assigned with any destination, it can merge with another group. To do so, group members from the smaller group change their membership to the bigger group. As a result of more mobile nodes joining into the bigger group, the resultant reference region, the size of which is proportional to the number of nodes in the group, is also enlarged (the details will be introduced in Section 3.2) as illustrated in Figure 3(b). Every mobile node from the smaller group randomly selects a point within the new reference region and moves towards it. When all mobile nodes from the smaller group have reached the larger group's reference region, the merger between these two groups is completed as shown in Figure 3(c).

It is easy to see that RRGM can be used to model the

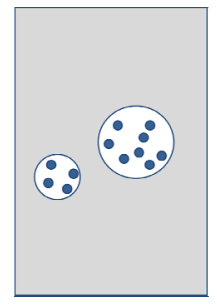

(a)

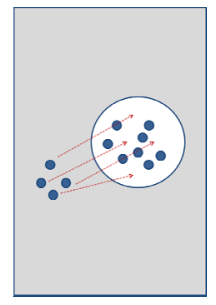

(b)

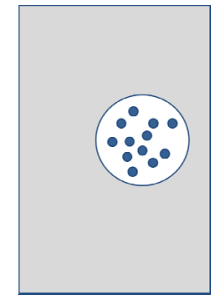

(c)
Figure 3. Merger of two groups. 
occurrence of group partitions and mergers in group mobility of MANETs. An example of such an event taking place is in the search and rescue operations. For such an operation, a rescue team may be assigned with several tasks simultaneously. As a result, some team members have to move apart from the original team that leads to a group partition. After a team of rescuers reached its destination and carried out tasks, they may merge with other teams.

\subsection{Model Configurations and Implementations}

Table 1 summarizes the input parameters used in RRGM and their default values. Their specific usages will be discussed along with the introduction of model implementations. Note as stated in Table 1, the reference region used could take the shape of either a rectangle or a circle, which is controlled by the parameter $\eta$. During implementation of the model, when $\eta=0$, it indicates that a circle is used as the shape of the reference region. Next, we give the definitions of some terms used in RRGM.

1) Center of a group/location of a group: it refers to the point whose coordinates are obtained by taking the average of the coordinates of all mobile nodes in the group.

Table 1. Input parameters of RRGM.

\begin{tabular}{|c|c|c|}
\hline \multicolumn{2}{|c|}{ Spatial parameters } & \multirow{2}{*}{$\begin{array}{l}\text { Default } \\
\text { values } \\
1000 \mathrm{~m}, \\
1000 \mathrm{~m} \\
(500, \\
500)\end{array}$} \\
\hline $\begin{array}{l}\text { T-length, } \\
\text { T-width } \\
\left(x_{0}, y_{0}\right)\end{array}$ & $\begin{array}{l}\text { Dimensions of the simulation area (terrain } \\
\text { size) in length and width (in meters). } \\
\text { Center's coordinates of the initial group. }\end{array}$ & \\
\hline \multicolumn{3}{|c|}{ Group and node related parameters } \\
\hline$N_{\text {total-node }}$ & $\begin{array}{l}\text { Total number of mobile nodes in the simu- } \\
\text { lation. }\end{array}$ & 50 \\
\hline$N_{\text {Initial-dest }}$ & Number of destinations initially generated. & 3 \\
\hline$N_{\text {standby-g }}$ & Standby groups initially deployed. & 0 \\
\hline$\rho$ & Density of nodes in a group. (nodes/100 $\mathrm{m}^{2}$ ) & 0.1 \\
\hline$v_{0}$ & Average node velocity. (m/s) & 10 \\
\hline$\gamma$ & Velocity coefficient. & 0.5 \\
\hline \multicolumn{3}{|c|}{ Timing parameters } \\
\hline$T$ & Simulation time. & $1000 \mathrm{~s}$ \\
\hline$T_{d}$ & Interval for generating new destinations. & $15 \mathrm{~s}$ \\
\hline$\tau_{0}$ & Pause time for a mobile node at a location. & $0 \mathrm{~s}$ \\
\hline$\tau_{1}$ & $\begin{array}{l}\text { Pause time for a reference region at a loca- } \\
\text { tion. }\end{array}$ & $2 \mathrm{~s}$ \\
\hline$\tau_{2}$ & Idle time for a group at a destination. & $10 \mathrm{~s}$ \\
\hline \multicolumn{3}{|c|}{ Miscellaneous } \\
\hline$\eta$ & $\begin{array}{l}\text { Ratio between the length and the width of a } \\
\text { reference region (for rectangular reference } \\
\text { region). If } \eta=0 \text {, it represents circular } \\
\text { reference region is used. }\end{array}$ & 1 \\
\hline$d_{x}, d_{y}$ & $\begin{array}{l}\text { Distance granularity for defining move- } \\
\text { ment trajectories of groups' in the range of } \\
(0,1) \text {. }\end{array}$ & $0.3,0.3$ \\
\hline$\delta$ & $\begin{array}{l}\text { A distance threshold between the center of } \\
\text { a group and a destination (in meters). }\end{array}$ & 10 \\
\hline $\begin{array}{l}\left(M_{x 1}, M_{y 1}\right) \\
\ldots, \\
\left(M_{x k}, M_{y k}\right)\end{array}$ & $\begin{array}{l}\text { Intermediate checkpoints of reference } \\
\text { regions (optional, used in scenarios with } \\
\text { predefined destinations). }\end{array}$ & n.a. \\
\hline
\end{tabular}

2) Center of a reference region/location of a reference region: it refers to the midpoint of a diagonal in the rectangle reference region, or the center of the circular reference region.

3) Distance between two groups: it refers to the distance between the centers of two groups.

Initially, all mobile nodes (i.e. $N_{\text {total-node }}$ nodes) are deployed in the simulation area as a single group. A reference region will be created at the center of the initial group, whose coordinates are $\left(x_{0}, y_{0}\right)$.

If a rectangle is used as the shape of the reference region, the length $l$ and width $w$ of the reference region are jointly determined by both $N_{\text {total-node }}$ and density of nodes in a group, namely $\rho$. Their relationship can be expressed by:

$$
N_{\text {total-node }}=\rho \times(l \times w)
$$

As the ratio between the length $l$, and the width $w$, of a reference region, namely $\eta$, is specified, where $\eta=l / w, l$ and $w$ can be calculated by:

$$
l=\sqrt{\frac{\eta \times N_{\text {total-node }}}{\rho}}, \quad w=\sqrt{\frac{N_{\text {total-node }}}{\rho \times \eta}}
$$

Note that the center of the reference region, whose coordinate is $\left(x_{0}, y_{0}\right)$, is the midpoint of a diagonal in the rectangle reference region as illustrated in Figure 4. So the range of the reference region would be from the bottom left $\left(x_{0}-l / 2, y_{0}-w / 2\right)$ to the top right $\left(x_{0}+l / 2, y_{0}+w / 2\right)$.

Similarly, if circle is considered as the shape of a reference region, the center of the circular reference region is $\left(x_{0}, y_{0}\right)$ and its radius $r$ can be calculated as:

$$
r=\sqrt{\frac{N_{\text {total-node }}}{\pi \times \rho}}
$$

Subsequently, $N_{\text {Initial-dest }}$ destinations are randomly generated. The initial group is logically partitioned into $N_{\text {Initial-dest }}+N_{\text {standby-g }}$ subgroups which are composed of $N_{\text {Initial-dest }}$ active subgroups (equivalent to the number of destinations) and $N_{\text {standby-g }}$ standby subgroups as specified in the configurations (Table 1). Standby groups are used

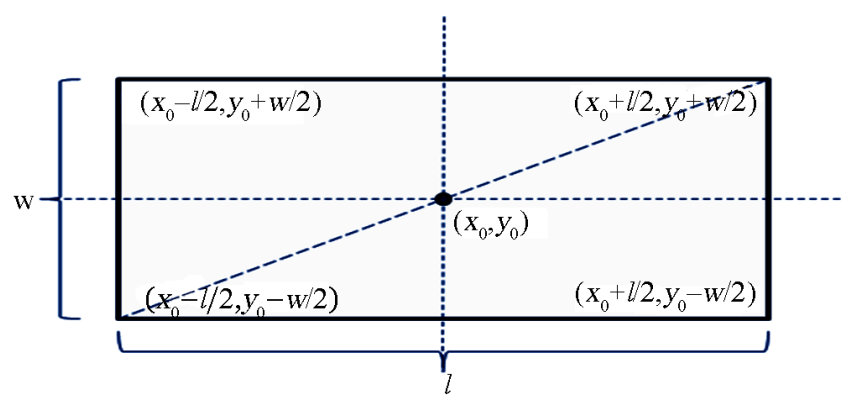

Figure 4. Illustration of a reference region. 
to model situations where groups do not have destinations to move towards. The default value of $N_{\text {standby-g }}$ in our implementation is zero. The destinations which have been generated are assigned to the active subgroups. As a result each active subgroup has one destination. For mobile nodes, each of them randomly selects a subgroup to join in.

Next, a reference region will be generated for each active subgroup. For a standby subgroup, the location of its reference region is placed at the center of the subgroup. The dimensions (the length and the width of a rectangle reference region, or the radius of a circular reference region) can be calculated using (2) or (3) respectively by replacing $N_{\text {total-node }}$ with the number of nodes in the subgroup. For an active subgroup, the location of its reference region can be calculated by the following steps:

Step 1: Given an active group $i$, let the coordinates of its destination be $\left(x_{d}, y_{d}\right)$, and the number of nodes in the subgroup be $N_{i}$.

Step 2: As it is already known that the coordinates of the initial group's location is $\left(x_{0}, y_{0}\right)$, we can calculate the location of the reference region of group $i$, namely $\left(x_{i}, y_{i}\right)$, by:

$$
\begin{gathered}
x_{i}=\left(x_{d}-x_{0}\right) d_{x} \times \operatorname{rand}()+x_{0} \\
y_{i}=\left(y_{d}-y_{0}\right) d_{y} \times \operatorname{rand}()+y_{0}
\end{gathered}
$$

where $\mathrm{d}_{x}$ and $\mathrm{d}_{y}$, in the range of $(0,1]$, are the distance granularity for defining the trajectory of a reference region to the destination. $\operatorname{rang}(\mathrm{)}$ is a random seed to generate a random number in the range of $(0,1)$ in order to avoid generating exactly same trajectory of a reference region to a destination by these equations.

Step 3: The dimensions (the length and the width of a rectangle reference region, or the radius of a circular reference region) of the reference region can be calculated using (2) or (3) respectively by replacing $N_{\text {total-node }}$ with $N_{i}$.

\subsection{Node's Movement and Displacement of a Reference Region}

Once a reference region is generated for a group, the respective group member will randomly select a point within the range of the reference region as its target for movement as stated in section 3.1. The velocity that each group member travels with, namely $v$, is varied by:

$$
v=\gamma \times v_{0}+\operatorname{rang}() \times v_{0}
$$

where $v_{0}$ is a pre-defined average velocity of mobile nodes, and the random seed rand () returns a random value within the range of $(0,1) . \gamma$ is a coefficient of $v_{0}$ and $\gamma \times v_{0}$ contributes a fixed value to $v$. Once a mobile node arrives at the point it has previously selected, it may pause for a period of time $\tau_{0}$ before continuing its movement by choosing a new point within the reference region as its target. The mobility of a mobile node within the range of its reference region is similar to that of the Random Waypoint Mobility in the sense that a mobile node travels with a selected speed towards a selected destination in each iteration. Practically, this can mimic the mobility of a team of rescuers carrying out operations in a small area before moving to a new disaster venue.

After all group members arrive at the reference region, the reference region may remain at its current location for a certain period, namely pause time of a reference region $\tau_{1}$, which is timed from the arrival of all group members to the reference region till a new location of the reference region is generated. Assuming that the current location of a reference region is $\left(x_{i}, y_{i}\right)$, its new location $\left(x_{i+1}, y_{i+1}\right)$ can be calculated by.

$$
\begin{aligned}
& x_{i+1}=\left(x_{d}-x_{i}\right) d_{x} \times \operatorname{rand}()+x_{i} \\
& y_{i+1}=\left(x_{d}-y_{i}\right) d_{y} \times \operatorname{rand}()+y_{i}
\end{aligned}
$$

The basic ideas of these two equations are as same as (4) and (5) respectively. By adjusting the values of $\mathrm{d}_{x}$ and $\mathrm{d}_{y}$, a group's movement trajectories could be different.

When the distance from the location of a reference region to its destination is smaller than a threshold $\delta$, it may be considered that the group has reached the destination, and the group may pause for duration $\tau_{2}$, namely group idle time, at this location. This ensures sufficient time for a group to move around the destination area to complete their tasks. After $\tau_{2}$, if there is no new destination assigned to this group, it becomes a standby group to indicate the completion of the task.

\subsection{Simulation of Mobility and Discussion}

Assuming that a reference region is stationary during the simulation (this can be realized by giving a big value to group partition interval), a node associated with this reference region will iteratively select a new target within the reference region for movement and this process will repeat until the simulation ends. The mechanism of this mobility pattern can be considered the same as that of Random Waypoint Mobility. In this section, simulations are conducted for each of these scenarios. The simulation is developed under $C++$ platform and visualized by NS2-nam [18].

In this subsection, we will present the generic group mobility operations of group partitions and mergers simulated by RRGM model, where destinations are generated periodically during the simulation. The parameters for the simulation setting are configured as in Table 2. 
Table 2. Input parameters for the simulation of generic group mobility pattern.

\begin{tabular}{lllll}
\hline$T$-length $=1000$ & $N_{\text {total-node }}=50$ & $\rho=0.1$ & $T_{d}=15$ & $\eta=0$ \\
\hline$T$-width $=1000$ & $N_{\text {Initial-dest }}=3$ & $v_{0}=10$ & $\tau_{0}=0$ & $d_{x}=0.3$ \\
$\left(x_{0}, y_{0}\right)=(500,0)$ & $N_{\text {standby-g }}=0$ & $\gamma=0.5$ & $\tau_{1}=2$ & $d_{y}=0.3$ \\
& & $T=1000$ & $\tau_{2}=10$ & $\delta=10$ \\
\hline
\end{tabular}

The size of simulation area is 1000 meters by 1000 meters for the length and the width respectively (i.e. $T$ - length $=1000$ and $T-$ width $=1000)$. Each simulation is run for 1000 seconds (i.e. $T=1000)$. 50 mobile nodes $\left(N_{\text {total-node }}=\right.$ $50)$ are deployed at $(500,0)$ initially $\left(\right.$ i.e. $\left(x_{0}, y_{0}\right)=$ $(500,0))$. 3 destinations (i.e. $N_{\text {Initial-dest }}=3$ ) are generated initially and no standby group will be generated (i.e. $N_{\text {standby-g }}=0$ ). Node density of each group is 0.1 node $/ 100 \mathrm{~m}^{2}$ (i.e. $\rho=0.1$ node $/ 100 \mathrm{~m}^{2}$ ). The average speed of mobile nodes is $10 \mathrm{~m} / \mathrm{s}$ (i.e. $v_{0}=10$ ) which is distributed in the range of $(5 \mathrm{~m} / \mathrm{s}, 15 \mathrm{~m} / \mathrm{s})$ derived according to (7) where the coefficient $\gamma$ is equal to 0.5. A new destination will be generated at every 15 seconds (i.e. $T_{d}=15$ ). A node will not pause during the simulation (i.e. $\tau_{0}=0$ ), and a reference region pauses for 2 seconds (i.e. $\tau_{1}=2$ which is timed from the arrival of all group members to the reference region till a new location is generated for this reference region). The group idle time $\tau_{2}$ is configured to 10 seconds. We use circle as the shape of reference regions in the simulation (i.e. $\eta=0$ ). The distance granularity for group's movement is 0.3 for both $d_{x}$ and $d_{x}$. When the distance between the center of a group and its destination is less than 10 meters (i.e. $\delta=10$ ), this group is considered to have already arrived to the destination.

Traces of nodes' movement are recorded and NS2Nam is used for visualization. Screenshots for mobile nodes in the network are captured to demonstrate their mobility. Figure 5 presents the occurrence of a group that is partitioned into several subgroups which subsequently merge. It is used to reflect group mobility related applications in MANETs, such as military operations in battlefields, disaster recovery and scientific explorations. Destinations can be used to represent enemy's bases in military operations, or destroyed sites in disaster recovery scenarios.

As shown in Figure 5(a), initially all nodes are deployed in a small area as the initial group. When three destinations D1, D2 and D3 are generated, the initial group is partitioned into three subgroups, A, B and C. Meanwhile, reference regions are also generated for each subgroup denoted by circles in the figures. Mobile nodes move towards their corresponding reference regions gradually. As shown in Figure 5(b) at the time $t=15 \mathrm{~s}$, a new destination D4 is generated. The closest subgroup

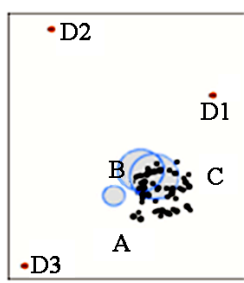

(a) $\mathbf{t}=0$

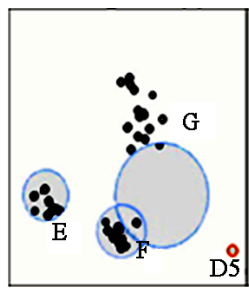

(d) $\mathrm{t}=77$

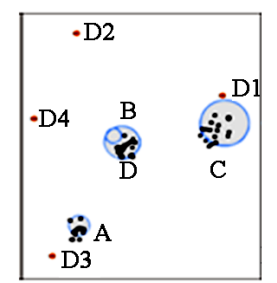

(b) $\mathrm{t}=15$

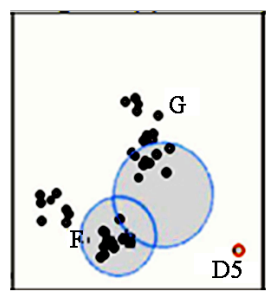

(e) $\mathrm{t}=82$

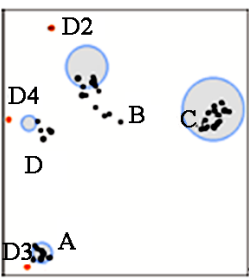

(c) $\mathrm{t}=20$

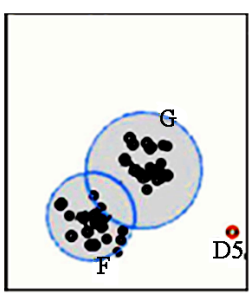

(f) $\mathbf{t}=85$
Figure 5. Generic group mobility pattern with group partitions and mergers.

B, which is moving towards D2, is split into two subgroups, $\mathrm{B}$ and $\mathrm{D}$, and the subgroup $\mathrm{D}$ heads towards the new destination D4. At $t=20 \mathrm{~s}$, the subgroup C arrived at D1 and it becomes a standby group, and D1 is removed as illustrated in Figure 5(c). From Figure 5(d) to Figure 5(f), screenshots for a group merger process are captured. In Figure 5(d), the two smaller subgroups E and $F$ are standby subgroups while the other subgroup $G$ is an active subgroup. In Figure 5(e), the reference region of the subgroup $\mathrm{E}$ is merged with subgroup F's reference region. The process of this group merger is completed at $t=85 \mathrm{~s}$ when all group members in $\mathrm{F}$ move into the reference region of subgroup $\mathrm{F}$ as shown in Figure 5 (f).

\section{Performance Evaluation of Network Routing Protocols Using RRGM}

In this section, we will evaluation the performance of AODV and DSR under the group mobility network environment generated by the RRGM model.

\subsection{Network Environment Configurations}

The simulation studies are carried out using the mobile network simulator QualNet [19]. To set up the simulation network environment, we generate 50 mobile nodes placed within a 1200 meters $\times 600$ meters grid (as elaborated in Table 3). Radio propagation range of each node is 250 meters with channel capacity of 2 Mbits/s. 20 sender-receiver pairs are designated randomly and each source can generate constant bit rate (CBR) traffic of 512 bytes data packet every second. Every simulation is run 
Table 3. Configurations of the network environment.

\begin{tabular}{ll}
\hline Parameters & Values \\
\hline Terrain size & 1200 meters $\times 600$ meters \\
Radio propagation range & 250 meters \\
Channel capacity & $2 \mathrm{Mbits} / \mathrm{s}$ \\
CBR traffic & 512 bytes/second \\
Simulation time & 900 second \\
Number of nodes & 50 \\
\hline
\end{tabular}

for 900 seconds. Each scenario is repeated for 20 times and the average values are finally presented in the simulation results. The simulation studies are carried out by varying the speeds of mobile nodes and node density ${ }^{1}$. Increase of speed can lead to fast change of network topology whereas in turn affects the network performance. Node density is used to describe the relative distance between group members. When the node density is low, it indicates that group members have a longer distance between one another, whereas high node density (e.g. a great many mobile nodes in a small area) means group members will be closed to each other. With a fixed number of mobile nodes in a group, reducing the node density will result in an increase in the average distance between group members. This in turn will result in an increase of the coverage area of the group. We note that the changes of group coverage will eventually have an impact on the network performance especially for a network where group partitions would take place frequently.

\subsubsection{Experimental Settings - Investigation on Speeds} This experiment is conducted according to two different scenarios, namely:

1) Scenario I-Group mobility with group partition disabled.

2) Scenario II-Group mobility with group partition enabled.

In Scenario I, group partitions are disabled by assigning a big value (which is greater than the simulation time) to the "partition interval" in RRGM. Therefore, group mergers would also not take place in this scenario. It is notable that due to the random mobility of groups, the transitory overlapping of groups is not considered as group mergers in this work. On the other hand, group partitions and mergers would take place in Scenario II by assigning a suitable value to the "partition interval" (e.g. every 40 seconds) in the RRGM model. Idle subgroups, which do not have any assigned destinations for movement, will merge with other subgroups.

In Scenario I, 50 nodes are initially deployed into three different subgroups such that each of the subgroup will consist of 16 or 17 group members. Since group

\footnotetext{
${ }^{1}$ Node density refers to the node density in a group, i.e. the number of nodes in an area where mobile nodes can move for their local movement in the group.
}

partitions are not allowed to take place in this scenario, the membership of each group is fixed throughout the simulation. We identify 20 source-destination pairs where $50 \%$ data packet transmission is made via inter-group communications (the source and destination pairs are placed in different subgroups respectively) and another $50 \%$ is via intra-group communications (the source and destination pairs are placed in the same group).

In Scenario II, 50 nodes are initially deployed all together in one group. Group partitions will take place in every 40 seconds interval with the RRGM model. However, if each subgroup has less than 10 nodes, it will not be further partitioned and newly generated destinations should be discarded. This is due to the fact that when group size ${ }^{2}$ is very small, there will be many small subgroups generated during the simulation and the communications will mostly be in the manner of inter-group communications. Hence, the experimental results will not lose the nature of intra-group communications.

The node density is fixed at 500 nodes $/ \mathrm{km}^{2}$ as configured in RRGM. We vary average node speeds from 1 $\mathrm{m} / \mathrm{s}$ to $20 \mathrm{~m} / \mathrm{s}$ to investigate its impact on the network performance. When the average node speed is high, more impetuous and arbitrary movement of groups will occur. Hence group partitions are expected to take place more frequently.

\subsubsection{Experimental Settings-Investigation on Node Densities}

In this experiment, node density is varied from 200 nodes $/ \mathrm{km}^{2}$ to 400 nodes $/ \mathrm{km}^{2}$ in steps of 50 nodes $/ \mathrm{km}^{2}$. Node speed is randomly generated within the range of $(15 \mathrm{~m} / \mathrm{s}, 25 \mathrm{~m} / \mathrm{s})$. Group partitions and mergers are enabled in this experiment. As discussed in the earlier part of this section, changing node density will result in the change of group's coverage area. However, we notice that most intra-group communications can be done via one hop. If group partitions and mergers do not take place, changes of node densities will not have a significant impact on the network performance. Hence, the scenario where group partitions and mergers are disabled is not considered for this experiment. The group partition interval is fixed at 40 seconds in RRGM. Other parameters are the same as those used in the experiment of investigating on node speeds as described in the subsection 4.1.1.

\subsection{Performance Metrics}

The performance metrics which will be used in the simulation include the packet delivery ratio $(P D R)$, the

\footnotetext{
${ }^{2}$ Group size is defined as the number of nodes in a group.
} 
average control packets per data packet sent $(A C P)$, and the end-to-end delay. $P D R$ reflects the percentage of data packets that can be successfully delivered, which is an important metric to evaluate the efficiency of a network. $A C P$ refers to the amount of routing packets required to set up and maintain routes in order to deliver data packets and it is then normalized by every data packet sent to indicate the average overhead spent in order to deliver a data packet. End-to-end delay measures the average time spent in the period when a packet is successfully sent from the source to the destination. Next, we will specifically introduce each of these performance metrics.

$P D R$ is calculated as the ratio of the number of data packets delivered to the destinations to those generated by the sources. Mathematically, it can be expressed as:

$$
P D R=\frac{\sum p_{\text {received }}}{\sum P_{\text {sent }}}
$$

$\sum p_{\text {received }}$ stands for the total number of data packets received at receivers, while $\sum P_{\text {sent }}$ is the total number of data packets sent during a simulation.

The control packets include route request (rreq), route reply initiated by intermediate nodes $(r r e p(1))$ and by destinations (rrep(2)) separately, and route error messages (rerr). We calculate the sum of all the control packets incurred during simulation, which is then normalized by the total number of data packet sent as represented by:

$$
A C P=\frac{\sum r r e q+\sum r r e p(1)+\sum r r e p(2)+\sum r e r r}{\sum P_{\text {sent }}}
$$

End-to-end delay refers to the time taken for a packet to be transmitted across a network from a source to its destination. This metric can reflect the quality of communications between users. This includes all possible delays caused by buffering during route discovery, queuing at the interface queue, retransmission delays at the MAC layer, propagation delay and transmission delay. Node's mobility may cause the breakage of established links which result in the loss of data packets and additional delay incurred as a result of packet retransmissions. The overall end-to-end delay can be defined as:

$$
E E D=\frac{1}{p_{\text {received }}} \sum_{i=1}^{p_{\text {received }}}\left(r_{i}-s_{i}\right)
$$

where $p_{\text {received }}$ is the number of successfully received packets at destinations, $i$ is the unique packet identifier, and $r_{i}$ is the time at which a packet with the unique id $i$ is received, while $s_{i}$ is the time at which a packet with the unique id $i$ is sent.

\subsection{Simulation Results of Routing Protocols}

\subsubsection{Varying the Average Speed}

Figure 6 and Figure 7 presents the results which show how the packet delivery ratio varies with mobility speeds. As illustrated in Figure 7, the trends of packet delivery ratio for both AODV and DSR increase as the average speed increases when group partition is disabled. The growth is $2 \%$ to $3 \%$ for both of AODV and DSR. Since $50 \%$ source-destination pairs are placed in different groups respectively for inter-group communications, they may not be connected initially if the sources can not find routes to their destinations, which may be due to the long distance beyond the transmission range or the lack of intermediate nodes between each other. When the network topology changes as nodes move faster, those previously disconnected source-destination pairs which are placed in different groups would possibly get connected. As a result, more data packets can be received by the destinations in inter-group communications. Hence, the packet delivery ratio is increased as the speed increases.

On the contrary, the performance of packet delivery ratio for both DSR and AODV falls dramatically under

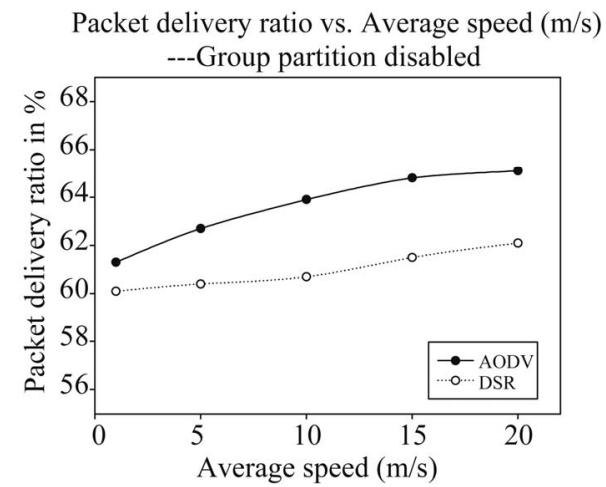

Figure 6. Packet delivery ratio vs. Average speed (m/s)Group partition disabled.

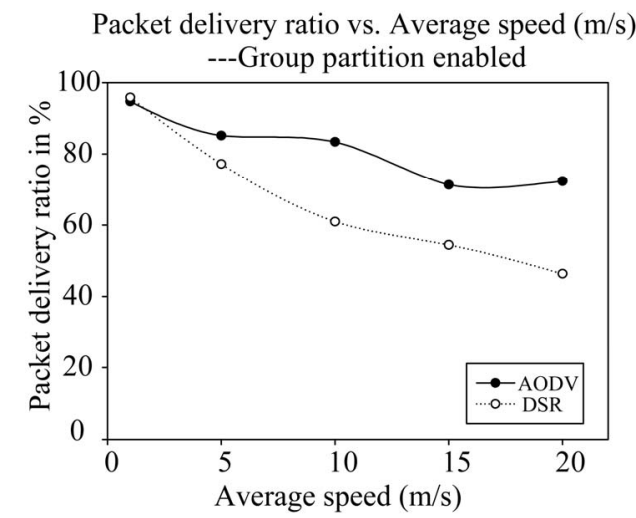

Figure 7. Packet delivery ratio vs. Average speed (m/s) Group partition enabled. 
the group partitioning scenario when nodes move faster as shown in Figure 7. A larger number of group partitions will occur when the node speed is increased. For example, a pair of source-destination previously in a same group would be easily separated into different groups when more group partitions take place and consequently data packets from the source can not be delivered to the destination. As a result, packet delivery ratio will drop, as reflected by the decreasing trend for both AODV and DSR respectively. It is notable that AODV still can retain a relatively high packet delivery ratio of $70 \%$ when the average speed is increased to $15 \mathrm{~m} / \mathrm{s}$ to $20 \mathrm{~m} / \mathrm{s}$. Comparatively, only $50 \%$ packets can be received in DSR at the speeds of $15 \mathrm{~m} / \mathrm{s}$ to $20 \mathrm{~m} / \mathrm{s}$. As discussed in subsection 2.2, AODV reacts faster than DSR when the network topology changes because AODV only keeps one route entry and when it becomes invalid, it will reestablish a new route. However, DSR has to maintain multiple routes entries before reinitiating a new route discovery process. Hence, AODV is more adaptive to frequent network topology changes than DSR and consequently it yields a higher packet delivery ratio than DSR. It is notable the packet delivery ratio for both AODV and DSR is up to $95 \%$ to $98 \%$ where DSR is slightly higher than AODV for about $1 \%$ when the speed is $1 \mathrm{~m} / \mathrm{s}$. As described in the earlier subsection 4.1.1, initially all mobile nodes are deployed in one big group for this scenario. When the speed is low, nodes barely move during the simulation (compared to the high speed cases). Hence, routes maintained by both AODV and DSR can be valid for longer period of time. Therefore, the packet delivery ratio is higher for low average speeds.

Comparing with Figure 6 and Figure 7, we notice that when group partition is enabled the packet delivery ratio drops very fast, which is due to the frequent changes of network topology. On the other hand, when group partition is disabled, the packet delivery ratio yielded from the intra-group communications will not change dramatically as a result the packet delivery ratio only varies in a small range from $60 \%$ to $65 \%$ as illustrated in Figure 6.

Figure 8 and Figure 9 present the results which show how average control packets by per packet sent varies with mobility speeds. In Figure 8 where group partition is disabled, the average control packets raises slightly as the speed increases and approximately in average 0.55 and 0.77 control packet is generated per data packet sent for AODV and DSR respectively. It tallies with the earlier discussion in this subsection that DSR uses more control overhead than AODV in route maintenance. The control packets are mainly consisted of two portions: control packets for inter-group communications and intra-group communications. When group partition is
Average control packet per pack sent vs. Average speed $(\mathrm{m} / \mathrm{s})$

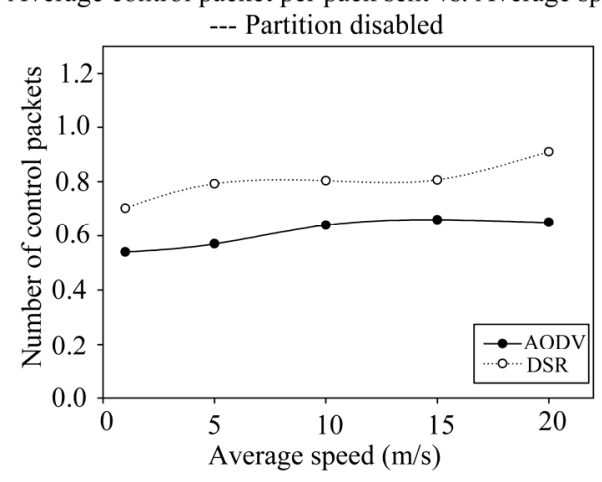

Figure 8. Average control packets per packet sent vs. Average speed-Group partition disable.

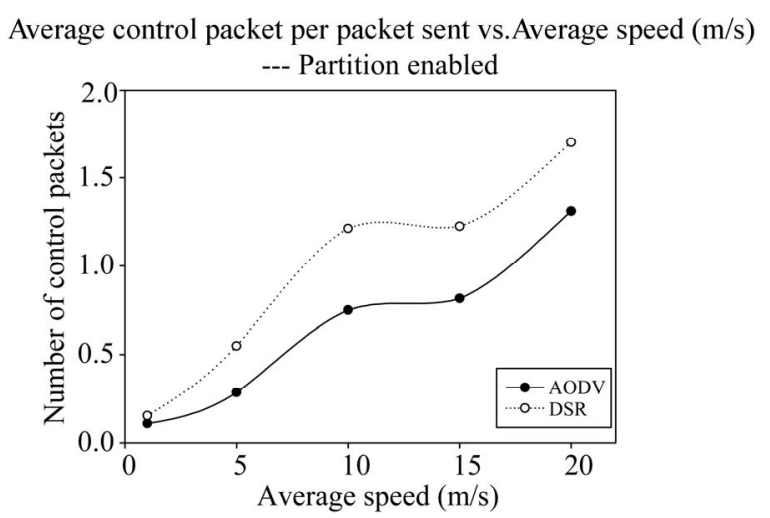

Figure 9. Average control packets per packet sent vs. Average speed-Group partition enabled.

disabled, the control packets for intra-group communications will be relatively stable since the group membership is not changed and hence routes can be valid for longer period of time during the simulation. On the other aspect, the control packets generated for inter-group communications will vary with the mobility speeds.when speed is increased, more control packets for route maintenance of inter-group communications will be generated, therefore, it results a slight increase in the number of control packets for AODV and DSR in this scenario.

In the group partition enabled scenario as displayed in Figure 9, more control packets are generated in both DSR and AODV when speed is raised. As initially, all mobile nodes are deployed together in one group, the intra-group commutations dominates at the beginning. It can be easily understood that intra-group communications are more stable than inter-group communications when group partitions do not occur. Therefore, the control packets are relatively low when the speed is as low as $1 \mathrm{~m} / \mathrm{s}$ (which we can say they barely move compared to the high speed cases). Correspondingly, it also can be reflected by a high packet delivery ratio as illustrated in Figure 7 when mobility speed is at $1 \mathrm{~m} / \mathrm{s}$. However, 
group partition takes place more frequently when the mobility speed increases as what we observed during the simulation. When group partitions take place, the previous stable intra-group communications would be disrupted, which results in massive route error messages and control packets for new route discovery process which would occur in both AODV and DSR. Thus, the control packets increase dramatically with the increase of mobility speeds as displayed in Figure $\mathbf{9}$.

The average control packets for the two scenarios as displayed in Figure 8 and Figure 9 also appear differently. The basic reason behind the trend of these simulation results is similar to what has been described for packet delivery ratio that group partitions lead to more topology changes which result more control packets generated for route maintenance.

Figure 10 and 11 illustrate the results of end-to-end delay of data packet delivery in group partition disabled and enabled scenarios respectively. In Figure 10, DSR generates much higher end-to-end delay than that of AODV, which is the same as what it does in Figure 11 (the end-to-end delay is small for both AODV and DSR when the speed the at $1 \mathrm{~m} / \mathrm{s}$ because all node are initially deployed in one group as discussed in section 4.1). The end-to-end delay in DSR is over 1.5 seconds whereas the end-to-end delay of AODV is only less than 0.3 second for both scenarios.

This great distinction in end-to-end delay between AODV and DSR is due to the difference of their working mechanisms. As introduce in subsection 2.2, caching is designed for keeping routing information in AODV and DSR. Caching is used in both route discovery process and route recovery process to increase the possibility of finding a route without initiating flooding of messages. In AODV, only one cache entry is allowed to be kept for each source-destination pair, while all possible routes are cached in DSR. In a dynamic environment, network topology changes very fast resulting in caching information becoming obsolete more quickly. Therefore, when a data packet is sent via a broken link in AODV, the node detecting the disconnection would return a route error message immediately and requests the source to reinitiate a new route discovery process. However, in DSR, the node detecting the route breakage sends a route error to the source node and the source would not reinitiate a new route discovery process until all route entries to the destination in its cache are tried.

It is possible for a source in DSR to keep multiple route entries via nodes in different groups to its destination. If a group, with which its intermediate nodes affiliate, has moved away, many of its route entries would become invalid simultaneously. The source node has to repair the broken links by trying all possible routes in its cache which results in a much higher end-to-end delay. On the contrary for AODV, as only one route entry is

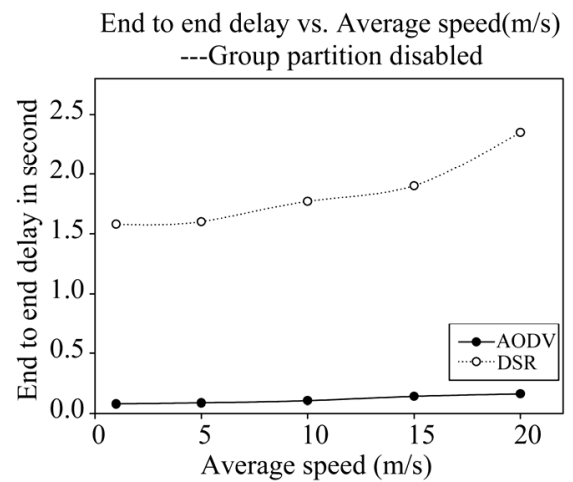

Figure 10. End-to-end delay vs. Average speed-Group partition disabled.

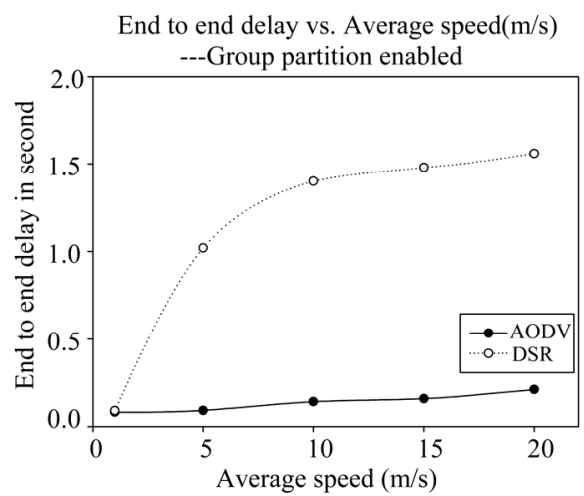

Figure 11. End-to-end delay vs. Average speed-Group partition enabled.

maintained by the source, the source can reinitiate a new route discovery process as soon as the route is no longer available. Therefore, the delay generated during the route recovery in DSR does not occur in AODV and thus the end-to-end delay in AODV is much lower than that of DSR as illustrated in Figure 10 and 11.

Comparing Figure 10 and 11, we notice that DSR generates lower end-to-end delay when group partition is enabled, which reduces from 1.7 seconds to 1.5 seconds approximately when the speed is up to $15 \mathrm{~m} / \mathrm{s}$. As the mobility speed increases, more group partitions would take place and with the aid of more subgroups, the source in DSR can find more alternative routes(via different subgroups) to the destinations. Therefore, the end-to-end delay in DSR can be reduced slightly. However, route entries in the sources in AODV become obsolete quickly when group partitions take place more frequently, and hence it yields a slightly higher end-to-end delay, which is about 0.05 second, in the partition enabled scenario.

\subsubsection{Varying the Node Density}

Figure 12 illustrates the changes in packet delivery ratio for AODV and DSR with respect to node densities. As it 
is shown, packet delivery ratio drops from $71 \%$ to $60 \%$ for AODV and from $68 \%$ to $54 \%$ for DSR with the node density increases from 200 nodes $/ \mathrm{km}^{2}$ to 400 nodes $/ \mathrm{km}^{2}$. AODV yields an approximate $10 \%$ higher packet delivery ratio than DSR. As discussed in the subsection 4.1.2, group coverage area is inversely proportional to the node density in the group. With a fixed number of mobile nodes, increasing node density in a group (where mobile nodes will be closer to other group members) will reduce the coverage area of the group. Therefore, links between connected groups may be broken because of the decrease of the group coverage areas which leads to a longer distance between those previously connected groups. As a result as illustrated in Figure 12, some data packets would not be delivered to the destinations via inter-group communications.

Figure 13 illustrates the changes of average control packets per data packet sent for AODV and DSR with respect to node densities. As the node density increases, group's coverage will be reduced as discussed in the subsection 4.1.2. As a result, less inter-group communications would be occurred and sources would not maintain as many route entries as that when more inter-group communications occur. Hence, the amount of maintenance control packets can be reduced when density is increased as shown in Figure 13. AODV uses nearly 50\% of the control packets that are used in DSR because AODV only keeps one route entry whereas DSR has to maintain multiple route entries which requires more control packets in route maintenance.

Figure 14 presents the results which show the end-to-end delay of AODV and DSR varied by node density. As discussed in the last subsection, when the node density increases, more links for inter-group communications may be broken (due to the coverage of groups become smaller which is inversely proportional to the node density). Therefore, more data packets would be lost which is illustrated in Figure 12 and retransmissions are required. Hence, more time will be spent on establishing new routes and it results in a higher end-to-end delay for both AODV and DSR. DSR generates a much higher end-to-end delay due to their different mechanisms node density increases, more links for inter-group communications may be broken (due to the coverage of groups become smaller which is inversely proportional to the node density). Therefore, more data packets would be lost which is illustrated in Figure 12 and retransmissions are required. Hence, more time will be spent on establishing new routes and it results in a higher end-to-end delay for both AODV and DSR. DSR generates a much higher end-to-end delay due to their different mechanisms to store and maintain route entries which has been discussed in the subsection 4.3.1.

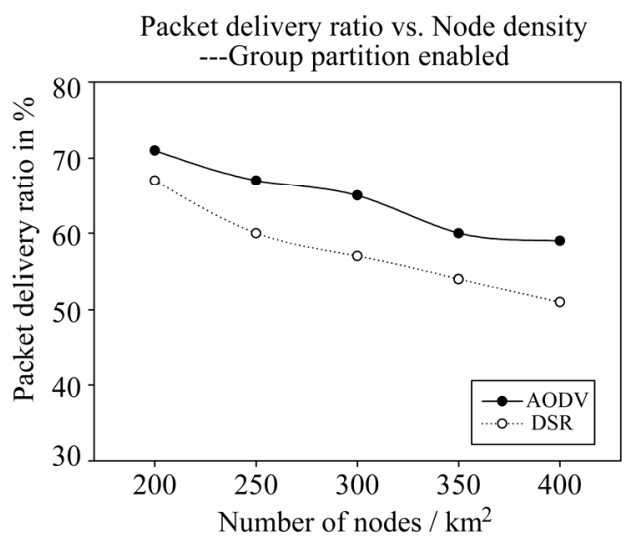

Figure 12. Packet delivery ratio vs. Node density-Group partition enabled.

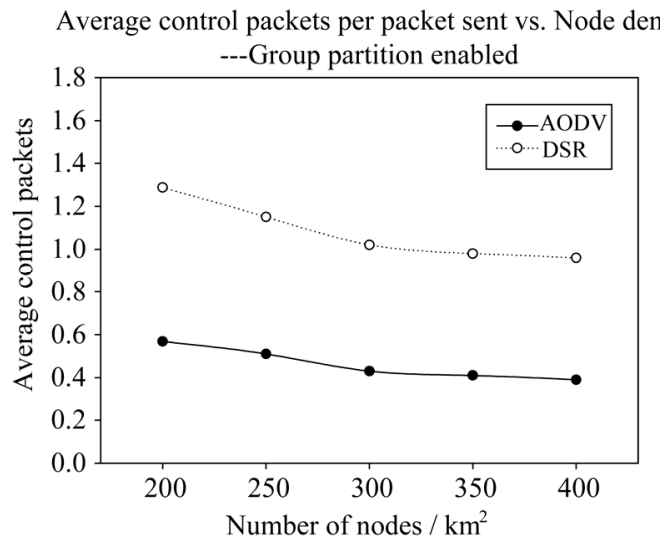

Figure 13. Average control packets per data packet sent vs. Node density-Group partition enabled.

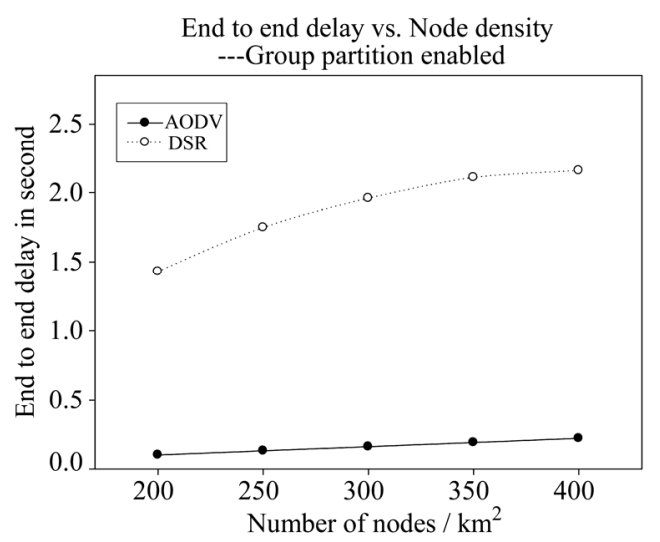

Figure 14. End-to-end delay vs. Node density-Group partition enabled.

\subsection{Discussion}

From the above comparisons and discussions, AODV shows its advantages in many aspects and outperforms DSR in group mobility network environment. The fun- 
damental difference between AODV and DSR is the mechanism of maintaining routing cache tables. AODV keeps only one route entry in its routing cache. Whenever the route is stale, it will reinitiate a new route recovery process. However, in DSR, multiple route entries are kept in each route table, if any breakage of a link is detected, a maintenance process has to be triggered to repair the broken links first before a new route discovery takes place. In dynamic network environment, routes turn stale quickly. Thus, more control packets are generated during the route recovery operations in DSR and therefore it has a longer delay. AODV is more adaptive to the dynamic environment and it is able to response quickly to link breakage by reconstructing a new route.

When a group partition takes place, some connections may be broken and communications between sources and destinations are interrupted. It results in more packet loss and packet delivery ratio is reduced. As almost every routing protocol is implemented with a route recovery mechanism, it takes more control packets to repair the broken links which may prolong end-to-end delay. High speed movement can greatly affect network performance. Increase of node density can also weaken network performance since it can change the coverage of groups which affect the relative distance between connected groups in the network. AODV has proven itself as a more efficient network routing protocol than DSR in group mobility environment of MANETs.

\section{Conclusions}

In this paper, we proposed RRGM model for simulation studies in MANETs which can be used to simulate group mobility. By taking the advantages of RRGM, group operations, such as partitions and mergers, can be realized. Simulations are conducted in modeling a number of different applications of ad hoc networks.

One step forward, we carried out a comprehensive study on the impact of group partitions and mergers to the network performance of two typical reactive routing protocols, AODV and DSR. The network mobility patterns are generated by RRGM model. AODV and DSR are compared by changing the speed and node density. Experiment results show that group partitions have a significant impact on the network performance which has never been revealed before. Frequent group partitions can downgrade the performance of both AODV and DSR. However, AODV shows its advantages in tackling with such kinds of group operations better than DSR. In addition, AODV is also more adaptive to high speed environment. On the other hand, DSR is suitable to a network with less mobility where the load of route maintenance is not heavy.

As a conclusion, it is impossible and not meaningful to find a pervasive routing protocol that can be adaptive to any network environment. Every network routing protocol may only work well under some particular network circumstance. Selecting a suitable network routing protocol is very important for studying the operations and performance of MANETs.

\section{References}

[1] F. Bai, N. Sadagopan and A. Helmy, "Important: A Framework to Systematically Analyze the Impact of Mobility on Performance of Routing Protocols for Ad Hoc Networks," IEEE Information Communications Conference (INFOCOM 2003), San Francisco, 30 March-3 April 2003, pp. 85-91.

[2] M. Sanchez and P. Manzoni, "ANEJOS: A Java Based Simulator for Ad Hoc Networks,” Future Generation Computer Systems, Vol. 17, No. 5, 2001, pp. 573-583.

[3] K. Pearson, "The Problem of the Random Walk," Nature, Vol. 72, No. 1, 1905, p. 294. doi:10.1038/072294b0

[4] T. Camp, J. Boleng and V. Davies, "A Survey of Mobility Models for Ad Hoc Network Research," Wireless Communication \& Mobile Computing (WCMC), Special issue on Mobile Ad Hoc Networking: Research, Trends and Applications, Vol. 2, No. 5, 2002, pp. 483-502.

[5] J. Broch, D. A. Maltz, D. B. Johnson, Y.-C. Hu and J. Jetcheva, "A Performance Comparison of Multi-Hop Wireless Ad Hoc Network Routing Protocols," The Fourth ACM Annual ACM/IEEE International Conference on Mobile Computing and Networking (Mobicom 98), Dallas, 25-30 October 1998, pp. 85-97.

[6] H. Moustafa and H. Labiod, "A Performance Comparison of Multicast Routing Protocols in Ad Hoc Networks," 14th IEEE Proceedings on Personal, Indoor and Mobile Radio Communications (PIMRC 2003), Beijing, 7-10 September 2003, pp. 497-501.

[7] S.-J. Lee, W. Su, J. Hsu, M. Gerla and R. Bagrodia, “A Performance Comparison Study of Ad Hoc Wireless Multicast Protocols,” The Nineteenth Annual Joint Conference of the IEEE Computer and Communications Societies (INFOCOM 2000), Tel Aviv, 26-30 March 2000, pp. 565-574.

[8] C. E. Perkins and E. M. Royer, “Ad-Hoc on-Demand Distance Vector Routing," Proceedings of the 2nd IEEE Workshop on Mobile Computing Systems and Applications, New Orleans, 25 February 1999, pp. 90-100.

[9] D. Johnson and D. Maltz, "Dynamic Source Routing in Ad Hoc Wireless Networks," Mobile Computing, Vol. 5, Kluwer Academic Publishers, Norwell, 1996, pp. 153-181.

[10] X. Hong, M. Gerla, G. Pei and C. Chiang, “A Group Mobility Model for Ad Hoc Wireless Networks,” ACM International Workshop on Modeling and Simulation of 
Wireless and Mobile Systems (MSWiM), Seattle, $20 \mathrm{Au}-$ gust 1999, pp. 53-60.

[11] M. Bouhorma, H. Bentaouit and A. Boudhir, "Performance Comparison of Ad-Hoc Routing Protocols AODV and DSR," International Conference on Multimedia Computing and Systems (ICMCS'09), Ouarzazate, 2-4 April 2009, pp. 511-514.

[12] E. M. Royer and T. Chai-Keong, “A Review of Current Routing Protocols for Ad Hoc Mobile Wireless Networks,” IEEE Personal Communications, Vol. 6, No. 2, 1999, pp. 46-55. doi:10.1109/98.760423

[13] O. S. Badarneh and M. Kadoch, "Multicast Routing Protocols in Mobile Ad Hoc Networks: A Comparative Survey and Taxonomy," EURASIP Journal on Wireless Communications and Networking, Vol. 2009, No. 1, 2009 , pp. 1-42. doi:10.1155/2009/764047

[14] S. Sesay, Z. K. Yang, B. Qi and J. H. He, "Simulation Comparison of Four Wireless Ad hoc Routing Protocols," Information Technology Journal, Vol. 3, No. 1, 2004, pp. 219-226.
[15] E. M. Royer and C.-K. Toh, "A Review of Current Routing Protocols for Ad Hoc Mobile Wireless Networks," IEEE Personal Communications, Vol. 6, No. 2, 2002, pp. 46-55. doi:10.1109/98.760423

[16] A. N. Ai-Khwildi, S. R. Chaudhry, Y. K. Casey and H. S. Ai-Raweshidy, "Mobility Factor in WLAN-Ad Hoc on-Demand Routing Protocols," IEEE 9th International Multitopic Conference (INMIC 2005), Karachi, 24-25 December 2005, pp. 1-6.

[17] M. Bansal and G. Barua, "Performance Comparison of Two on-Demand Routing Protocols for Mobile Ad Hoc Networks,” 2002 IEEE International Conference on Personal Wireless Communications, New Delhi, 15-17 December 2002, pp. 206-210. doi:10.1109/ICPWC.2002.1177278

[18] NS2-Nam, 2010. http://www.isi.edu/nsnam/ns/

[19] Scalable Network Technologies, 2010. www.scalablenetworks.com 